\title{
Semiclassical description of resonance-assisted tunneling in one-dimensional integrable models
}

\author{
Jérémy Le Deunff, ${ }^{1}$ Amaury Mouchet, ${ }^{2}$ and Peter Schlagheck ${ }^{3}$ \\ 1 Max-Planck-Institut für Physik komplexer Systeme, \\ Nöthnitzer Straße 38, 01187 Dresden, Germany. \\ 2 Laboratoire de Mathématiques et Physique Théorique, \\ Université François Rabelais de Tours — CNRS (UMR 7350), \\ Fédération Denis Poisson, Parc de Grandmont, 37200 Tours, France. \\ 3 Département de Physique, University de Liege, 4000 Liège, Belgium.
}

(Dated: August 3, 2021)

\begin{abstract}
Resonance-assisted tunneling is investigated within the framework of one-dimensional integrable systems. We present a systematic recipe, based on Hamiltonian normal forms, to construct onedimensional integrable models that exhibit resonance island chain structures with accurately controlled sizes and positions of the islands. Using complex classical trajectories that evolve along suitably defined paths in the complex time domain, we construct a semiclassical theory of the resonance-assisted tunneling process. This semiclassical approach yields a compact analytical expression for tunneling-induced level splittings which is found to be in very good agreement with the exact splittings obtained through numerical diagonalisation.
\end{abstract}

PACS numbers: 03.65.Sq, 03.65.Xp, 05.60.Gg, 05.45.Mt, Keywords:

\section{INTRODUCTION}

In quantum theory, the term tunneling defines classically forbidden processes - i.e. which cannot be described by real solutions of Hamilton's equations of motion - and was originally employed to characterize transitions which were forbidden by energy barriers. It has been thereafter extended to dynamical tunneling which refers to any quantum transition between two classically distinct regions in phase space [1, 2] where the inhibition of a classical transition between these two regions is not necessarily restricted to the constraint of energy conservation. Indeed, focusing first on the simple case of integrable systems with one degree of freedom, the development of semiclassical techniques [3] have permitted a deeper qualitative and quantitative understanding of tunneling in terms of classical trajectories. In particular, its complete semiclassical description requires, in addition to real orbits, to take into account also complex classical trajectories [4, 5]. For instance, studying scattering phenomena involved in chemical reactions, Freed [6] and George and Miller [7, 8] incorporated complex orbits, evolving along suitable paths in the complex time domain, in order to compute the Green function $G\left(q_{f}, q_{i}, E\right)$ giving rise to the tunneling transmission with an energy $E$ below the top of a potential barrier.

A few years later, Coleman [9] (see also Ref. [10]) developed an approach suited for the simplest bounded systems, where tunneling is generally identified in the spectrum as small splittings between doublets of nearly degenerate discrete eigenenergies. In the context of field theories, he introduced the notion of instantons which corresponds to classical solutions of the Hamilton dynamics once a Wick rotation $t \rightarrow-$ it has been performed. For systems with the standard form of the Hamiltonian

$$
H(p, q)=\frac{p^{2}}{2}+V(q)
$$

where $p$ and $q$ are the canonical variables, this transformation on the time leads to an inversion of the potential $V(q) \rightarrow-V(q)$. The classical trajectories in the new potential allow to evaluate quantum observables associated with the lowest energies (ground-state doublet or multiplet), such as the frequency of oscillation between an arbitrary number of identical minima, or the decay rate of a metastable state that is initially defined in a local minimum of the potential and decays via the coupling to a continuum of unbounded states.

The method has been recently generalized [11], using again the idea of a suitably parametrized complex time path, in order to embrace more general situations involving, e.g., an arbitrary energy and/or Hamiltonians not necessary of the form (11). For instance, resonant tunneling, which has been widely investigated in onedimensional (1D) open systems with two consecutive barriers [12 14], is thus explained in terms of constructively interfering repetitions of complex orbits. This is shown for the simple case of a triple-well potential where the presence of a deeper middle well (which prevents the application of the standard instanton techniques based on the complete Wick rotation recalled above) can create giant fluctuations of the tunneling period between the two symmetric outer wells [15], namely whenever a third energy level, associated with a state that is localized in this middle well, comes close to a doublet that is associated with the two outer wells.

If the number of the degrees of freedom exceeds 1, we generically deal with nonintegrable Hamiltonians whose phase space contains regular islands foliated with 
Kolmogorov-Arnold-Moser (KAM) tori and surrounded by chaotic seas. In that case, tunneling is drastically modified and yields erratic fluctuations (by several orders of magnitude) of the associated rates and time scales when varying a parameter of the system [16, 17]. These fluctuations have the same origin in the quantum spectrum as the ones observed in the one-dimensional resonant case. However, the appearance of natural boundaries [18] prevents the analytical continuation of the invariant classical KAM tori into the complex plane of the classical phase space, and the methods at work for one-dimensional systems fail. Despite some important breakthroughs in that direction during the past few decades [19 21], a full semiclassical description in terms of complex classical structures is still missing. On the other hand, a considerable effort has been made to combine, within a perturbative framework, statistical descriptions of chaos-assisted tunneling due to the influence of the classical chaotic sea 21 23 with the theory of resonance-assisted tunneling (RAT) [24 29] that is based on the presence of nonlinear resonances within the regular regions. This approach has been shown to provide rather accurate semiclassical predictions of quantum tunneling rates in kicked model systems [29].

In this paper, we present and discuss a semiclassical formula for resonance-assisted tunneling splittings in one dimensional integrable systems that exhibit a pair of symmetric regions of bounded motion in the classical phase space, each of them being surrounded by a resonant island chain. The study of such models is clearly inspired by the recent idea to mimic regular regions of mixed systems with a fictitious integrable approximation in order to predict regular-to-chaotic tunneling [30, 31], although we are not aiming here at approximating a given nonintegrable system by such a model. Instead, our motivation is to obtain a fully semiclassical (and nonperturbative) description of resonance-assisted tunneling through the analytical continuation of invariant classical manifolds to the complex domain, which is permitted by the integrability of the Hamiltonian. This will allow us to understand how the island chains in the phase space are at work to create fluctuations in the tunneling-induced level splittings when varying a parameter of the system and what the semiclassical conditions are for resonant tunneling.

The paper is organized as follows. In Sec. III, we construct a class of models that fulfill the classical properties mentioned above using the theory of Hamiltonian normal forms. Sec. III is dedicated to the computation of tunneling splittings. In Sec. IIIB, we shall investigate the complex manifold of our integrable model and identify the relevant complex classical trajectories, defined along well-suited complex time paths, that give rise to a semiclassical formula [Eq. (21) below] for resonance-enhanced level splittings. The perturbative RAT method is then applied to our system in Sec. IIIC and compared with the complex paths approach. We discuss the validity of the two methods in both limits of small and large sizes of the island chains.

\section{THE MODEL}

\section{A. Normal form theory}

The Hamiltonian normal forms in classical mechanics 32, 33. have been originally developed by Birkhoff 34. and extended by Gustavson [35] with the aim to classify the classical dynamics in the neighborhood of the periodic orbits in nonintegrable systems with several degrees of freedom. This classification relies on canonical equivalence and provides the simplest (local) form of the Hamiltonian where the only terms that are kept are those that are sufficient to supply the intrinsic "skeleton" of the dynamics, i.e., those terms that cannot be eliminated by a canonical transformation because they genuinely encapsulate the geometrical features of the dynamics. Hamiltonian normal forms have helped to predict the quantum energy spectra of such systems [36, 37].

Normal form approaches are based on the combination of Fourier and Taylor expansions of the nonintegrable Hamiltonian in the neighbourhood of a periodic orbit. Order by order, beyond the quadratic terms, a sequence of canonical transformations can be explicitly built to eliminate all terms but the resonant ones. The latter may give rise to divergencies manifesting in the above construction procedure, which is well known as the problem of small denominators. Those resonant terms inhibit the integration of systems of ordinary differential equations, and generally the procedure to obtain an accurate approximation of the dynamics does not converge, which is a signature of the non integrability of the system. Nevertheless, this procedure enables one to extract some essential information about the fine structure of the phase space as it provides a description not only of the regular part but also of the resonant layout where chaos emerges from, thereby leading to the simplest local integrable approximation of the system.

Specifically, let us consider an autonomous system with two degrees of freedom (or a periodically time-dependent system with one degree of freedom) depending on one control parameter $\epsilon$. In the neighborhood of a nondegenerate stable orbit, a transverse coordinate system $(p, q)$ in the so-called Poincaré surface of section can be chosen [38] such that the transverse dynamics is governed by a Hamiltonian whose normal form is given by (see Ref. [38] for an exhaustive and systematic study on this matter)

$$
\begin{gathered}
h^{(\ell)}(p, q ; \epsilon)=\frac{\omega(\epsilon)}{2}\left(p^{2}+q^{2}\right)+\sum_{k=2}^{\lfloor\ell / 2\rfloor} a_{k}(\epsilon)\left(p^{2}+q^{2}\right)^{k} \\
+b_{\ell}(\epsilon) \operatorname{Re}\left[(p+\mathrm{i} q)^{\ell}\right]+\text { higher order terms }
\end{gathered}
$$

where $\omega,\left\{a_{k}\right\}$ and $b_{\ell}$ are real parameters, $\lfloor\cdot\rfloor$ denotes the integer part, and the index $\ell \geq 3$ represents the order of 
the first angle-dependent resonant term occurring in the expansion. It can be rewritten in terms of the actionangle variables $I=\left(p^{2}+q^{2}\right) / 2$ and $\theta=\tan ^{-1}(q / p)$ associated with the one-dimensional harmonic oscillator,

$$
\begin{aligned}
& \tilde{h}^{(\ell)}(I, \theta ; \epsilon)=\omega(\epsilon) I+\sum_{k=2}^{\lfloor\ell / 2\rfloor} a_{k}(\epsilon)(2 I)^{k} \\
& \quad+b_{\ell}(\epsilon)(2 I)^{\ell / 2} \cos (\ell \theta)+\text { higher order terms } .
\end{aligned}
$$

It is straightforward to see that the resonant term creates, for appropriate values of the parameters, an island chain of $\ell$ islands in the transverse dynamics around the origin $(p, q)=(0,0)$ where the periodic orbit of the 2D system intersects the Poincaré surface of section.

\section{B. The Hamiltonian}

Let us rewrite the normal form (2) as

$$
h^{(\ell)}(p, q) \stackrel{\text { def }}{=} h_{0}^{(\ell)}\left(p^{2}+q^{2}\right)+v^{(\ell)}(p, q)
$$

with

$$
\begin{aligned}
h_{0}^{(\ell)}(I) & \stackrel{\text { def }}{=} a_{1} I+\sum_{k=2}^{\lfloor\ell / 2\rfloor} a_{k}(2 I)^{k}, \\
v^{(\ell)}(p, q) & \stackrel{\text { def }}{=} \operatorname{Re}\left[b(p+\mathrm{i} q)^{\ell}\right],
\end{aligned}
$$

where the $\left\{a_{k}\right\}$ are real parameters and $b \equiv|b| \exp (\mathrm{i} \phi)$ is a complex parameter. From now on, we consider $h_{0}^{(\ell)}$ to be the unperturbed part while $v^{(\ell)}$ has to be understood as a perturbation. Starting from $h^{(\ell)}$, we perform the substitution $(p, q) \mapsto(\cos p, \cos q)$ and thereby obtain the new Hamiltonian,

$$
H^{(\ell)}(p, q) \stackrel{\text { def }}{=} h^{(\ell)}(\cos p, \cos q)
$$

with which we shall work from now on. This construction gives rise to a smooth periodic replication of the phase space structure in both position and momentum. We then restrict $H^{(\ell)}(p, q)$ to the torus $[-\pi, \pi] \times[-\pi, \pi]$ by imposing $2 \pi$-periodic boundary conditions of the wave function in both $p$ and $q$. For a suitable choice of $\left\{a_{k}\right\}$ and $b$, this torus encloses four elementary cells centered at $(p, q)=( \pm \pi / 2, \pm \pi / 2)$ and surrounded by a $(\ell: 1)$ resonance chain, as illustrated in Fig. 2. While the modulus $|b|$ controls the size of the island chains, we can tune the relative orientation of the main islands by smoothly rotating the $(\ell: 1)$ resonances via the phase $\phi$ (see Fig. (2).

Focusing now on the simplest case $\ell=4$ [53], we obtain a model with $(4: 1)$ resonances:

$$
\begin{aligned}
h(p, q) & \stackrel{\text { def }}{=} h^{(4)}(p, q), \\
& =\frac{a_{1}}{2}\left(p^{2}+q^{2}\right)+a_{2}\left(p^{2}+q^{2}\right)^{2}+\operatorname{Re}\left[b(p+\mathrm{i} q)^{4}\right] .
\end{aligned}
$$

a)

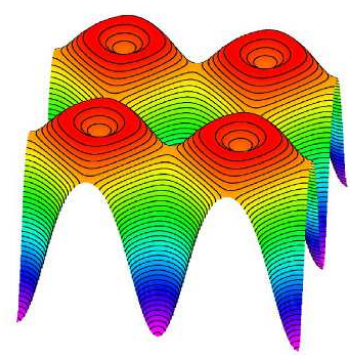

$H(p, q)$

b)

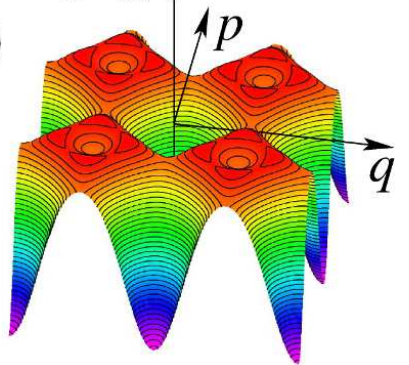

Figure 1: (Color online) Graph of $(p, q) \mapsto H(p, q)$ on the fundamental domain $[-\pi, \pi] \times[-\pi, \pi]$ where $H$ is given by Eq. (8) with $a_{1}=1, a_{2}=-0.55, \phi=0$ and (a) $b=0$; (b) $|b|=0.05$.

The Hamiltonian that we shall work with is given by

$$
H(p, q) \stackrel{\text { def }}{=} H^{(4)}(p, q)=H_{0}(p, q)+V(p, q),
$$

with

$$
\begin{array}{r}
H_{0}(p, q)=\frac{a_{1}}{2}\left(\cos ^{2} p+\cos ^{2} q\right)+a_{2}\left(\cos ^{2} p+\cos ^{2} q\right)^{2} \\
V(p, q)=|b|\left\{\left(\cos ^{4} p+\cos ^{4} q-6 \cos ^{2} p \cos ^{2} q\right) \cos \phi\right. \\
\left.-4\left(\cos ^{3} p \cos q-\cos ^{3} q \cos p\right) \sin \phi\right\}(8 \mathrm{c})
\end{array}
$$

Choosing $a_{1}>0$ and $a_{2}<0$, the energy profile $(p, q) \mapsto$ $H(p, q)$ exhibits four symmetric volcano-like patterns within the torus $[-\pi, \pi] \times[-\pi, \pi]$, each one having a local minimum located at the center $( \pm \pi / 2, \pm \pi / 2)$ of the corresponding elementary cell and four identical maxima situated along the crown of the volcanos (see Fig. 1).

\section{TUNNELING SPLITTINGS}

\section{A. Quantum mechanics}

For bounded Hamiltonians $H(p, q)$ with a twofold symmetry, the spectrum is made of discrete energies $E_{n}^{ \pm}$ which can be classified according to their parity $( \pm)$. They are determined from the stationary Schrödinger equation

$$
H(\hat{p}, \hat{q})\left|\phi_{n}^{ \pm}\right\rangle=E_{n}^{ \pm}\left|\phi_{n}^{ \pm}\right\rangle
$$

(with $\hat{p}$ and $\hat{q}$ denoting the momentum and position operator, respectively) where the natural integer $n$ sorts the corresponding eigenstates $\left|\phi_{n}^{ \pm}\right\rangle$which form an orthonormal basis. In the limit $\hbar \rightarrow 0$ (when Planck's constant is much smaller than the classical phase-space areas), the association of the quantum states $\left|\phi_{n}^{ \pm}\right\rangle$with classical phase-space structures can be visualized using the semiclassical Wigner or Husimi distributions 39 41]. In the case of regular classical dynamics, the states are 
a) $\phi=0$

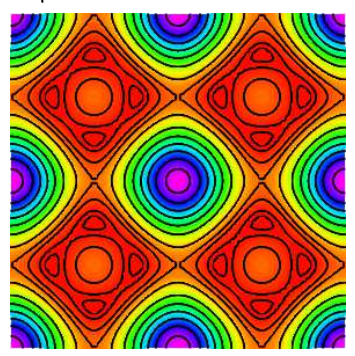

c)

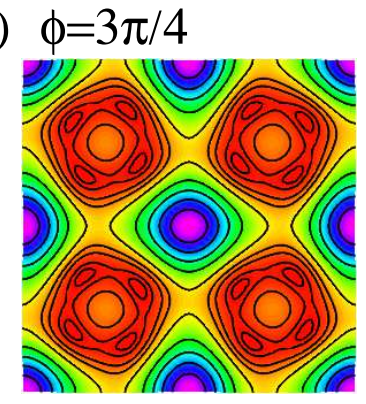

b) $\phi=\pi / 2$
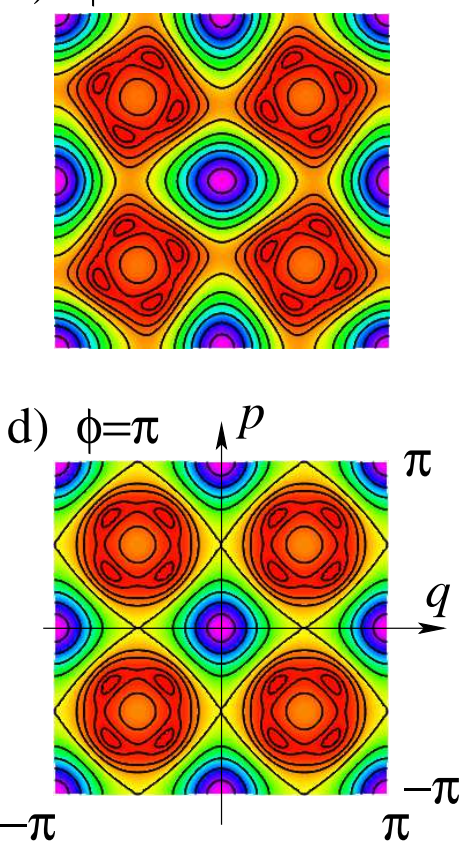

d) $\phi=\pi \quad p$

Figure 2: (Color online) Phase space of the Hamiltonian (8) with $a_{1}=1, a_{2}=-0.55,|b|=0.05$ for different values of the phase (a) $\phi=0$, (b) $\phi=\pi / 4$, (c) $\phi=\pi / 2$, (d) $\phi=\pi$.

sharply localized [up to order $\mathrm{O}(\hbar)$ ] along invariant tori in the classical phase space.

For instance, in the simple case of a 1D Hamiltonian of the standard form (1) with a potential $V(q)$ that exhibits two symmetric local minima, the eigenstates $\left|\phi_{n}^{ \pm}\right\rangle$with energies $E_{n}^{ \pm}$below the top of the barrier between the minima are mainly localized on symmetric tori in the wells characterized by the classical energy $E_{n} \simeq E_{n}^{+} \simeq E_{n}^{-}$. A quantum state that is given by the symmetric or antisymmetric linear combination of the eigenstates $\left|\phi_{n}^{ \pm}\right\rangle$is no longer stationary but gives rise to oscillations from one well to the other with the period $\tau=2 \pi \hbar / \Delta E_{n}$, where $\Delta E_{n} \stackrel{\text { def }}{=}\left|E_{n}^{-}-E_{n}^{+}\right|$is the splitting of the levels $E_{n}^{ \pm}$in the spectrum. This splitting represents, for both integrable and nonintegrable bounded systems, a characteristic signature of tunneling between two classically separated regions in the phase space.

The Hamiltonian $\hat{H}$ of our quantum model is derived from a straightforward quantization of the classical model (8) with the simple symmetrization rule

$$
f(p) g(q) \mapsto \frac{1}{2}[f(\hat{p}) g(\hat{q})+g(\hat{q}) f(\hat{p})]
$$

for the product of two functions $f(p)$ and $g(q)$ [42, 43]. From the quantization of (8), the periodicity of the Hamiltonian allows us to use the Floquet-Bloch theorem and to restrict, for integer values of $2 \pi / \hbar$, the analysis to the finite-dimensional Hilbert space $\mathcal{H}$ spanned by strictly periodic eigenstates in both position and momentum on the torus $[-\pi, \pi] \times[-\pi, \pi]$.
As we see in Fig. 2 two independent twofold symmetries are relevant in our model. It is natural to associate these two symmetries with the antiunitary operators $\hat{\Pi}_{q}$ and $\hat{\Pi}_{p}$ that perform mirror operations with respect to the $p$ and $q$ axes, respectively, and that are defined through $\hat{\Pi}_{q} f(\hat{p}, \hat{q}) \hat{\Pi}_{q}=f(\hat{p},-\hat{q})$ and $\hat{\Pi}_{p} f(\hat{p}, \hat{q}) \hat{\Pi}_{p}=$ $f(-\hat{p}, \hat{q})$ for any function $f$ of the canonical operators $\hat{p}$ and $\hat{q}$. Obviously, $\hat{\Pi}_{p}$ is the standard time-reversal operator, while $\hat{\Pi}_{q}$ is the time-reversal operator composed with the usual unitary parity operator. By construction, $\hat{\Pi}_{q}$, $\hat{\Pi}_{p}$, and $\hat{H}$ mutually commute with each other. However, the time-reversal invariance of the Hamiltonian cannot be exploited to discriminate among its eigenstates; it only allows one to choose the latter to be entirely real. This particular phase convention fixes the spectrum of $\hat{\Pi}_{q}$ to be identical to the spectrum of the parity operator, such that we can classify the eigenstates of the Hamiltonian according to their parity: $\hat{\Pi}_{q}\left|\phi_{n}^{ \pm}\right\rangle= \pm\left|\phi_{n}^{ \pm}\right\rangle$.

In contrast to conventional double-well systems, however, the eigenenergies associated with the four main islands within the unit cell are organized in quartets, and the parity alone is not sufficient to unambiguously specify the doublet whose level splitting is determined by tunneling along, say, the $q$ direction. To lift this ambiguity, we numerically construct four states $| \pm, \pm\rangle$ from the local $n$ th excited harmonic oscillator eigenstates $\mid \operatorname{Ror} L, U$ or $D\rangle$ that are centered at $(q, p)=( \pm \pi / 2, \pm \pi / 2)$ [with $L(R)$ referring to the left (right) column and $U(D)$ to the upper (lower) row within the unit cell depicted in Fig. 2, namely through

$$
\begin{aligned}
& |++\rangle \stackrel{\text { def }}{=} \frac{1}{2}(|R U\rangle+|L U\rangle+|L D\rangle+|R D\rangle) ; \\
& |-+\rangle \stackrel{\text { def }}{=} \frac{1}{2}(|R U\rangle-|L U\rangle-|L D\rangle+|R D\rangle) ; \\
& |+-\rangle \stackrel{\text { def }}{=} \frac{\mathrm{i}}{2}(|R U\rangle+|L U\rangle-|L D\rangle-|R D\rangle) ; \\
& |--\rangle \stackrel{\text { def }}{=} \frac{\mathrm{i}}{2}(|R U\rangle-|L U\rangle+|L D\rangle-|R D\rangle)
\end{aligned}
$$

Being eigenstates of the parity operator (with the eigenvalues 1 for $|++\rangle$ and $|--\rangle$ and -1 for $|+-\rangle$ and $|-+\rangle$ ) [54], these states closely mimic the quartet of eigenstates of the Hamiltonian that are localized within the centers of the four islands. In order to focus on tunneling along the $q$ direction, we therefore select those two eigenstates of $\hat{H}$ that have a maximal numerical overlap with $|++\rangle$ and $|-+\rangle$.

Because of the invariance of $H(\hat{p}, \hat{q})$ under the canonical transformation $(\hat{p}, \hat{q}) \mapsto(\hat{q},-\hat{p})$, tunneling along the momentum direction will give rise to the same splittings as for the position direction. This equivalence is reflected by an exact degeneracy between two energies among the four of the quartet: For even $N / 4$ the two states having a maximal overlap with $|+-\rangle$ and $|-+\rangle$ have exactly the same energies, while for odd $N / 4$ the levels corresponding to $|++\rangle$ and $|--\rangle$ are exactly degenerate. 


\section{B. Semiclassical theory}

By construction of the Hamiltonian (8a), the unperturbed case $b=0$ gives rise to a tunneling problem that is equivalent to the one of a symmetric 1D double-well system. This scenario has been intensively investigated using JWKB analysis in order to connect two approximated eigenstates, the so-called quasimodes, each of them being localized on a distinct real torus [44, 45]. Up to a prefactor of order 1 , the level splitting $\Delta E_{n}$ associated with the doublet at energy $E_{n}$ is essentially determined as [46, 47]

$$
\Delta E_{n} \underset{\hbar \rightarrow 0}{\sim} \hbar \omega_{n} \mathrm{e}^{-\Sigma\left(E_{n}\right) /(2 \hbar)}
$$

where $\omega_{n}$ is the frequency of classical oscillation on the torus with energy $E_{n}$ within the left or right well, and $\Sigma\left(E_{n}\right)$ is the imaginary action of a closed complex path that connects the two symmetric tori.

The simplest bounded model inducing quantum resonances can be obtained [15] for a Hamiltonian of the form (11) with a potential $V$ that has three wells, two symmetric outer ones, say, that are separated by a deeper central well. In such a system, resonant tunneling arises due to the constructive interference of classical paths that are bouncing back and forth between the two tunneling barriers. It was shown in Ref. [11] that these relevant orbits can be obtained from a suitable complex time path $s \mapsto \operatorname{Re} t(s)+\mathrm{i} \operatorname{Im} t(s)$ where, unlike for a pure Wick rotation, both the real and the imaginary part of the complex time are necessary to concatenate the primitive orbits that constitute the complex trajectories. For the resonance-assisted tunneling problem presented by the model (8) with $b \neq 0$, we shall, in the same spirit, introduce a generic type of concatenated complex paths that connect two real symmetric tori inside the main island of each cell. This set of orbits will be used to predict tunneling splittings between states that are localized within these islands. For a given real parametrization $s \mapsto t(s)$ of a complex time path, it can be easily shown [11, $\S$ III.A], using the analyticity of the Hamiltonian $H$ with respect to $(p, q, t)$, that the complex Hamiltonian equations

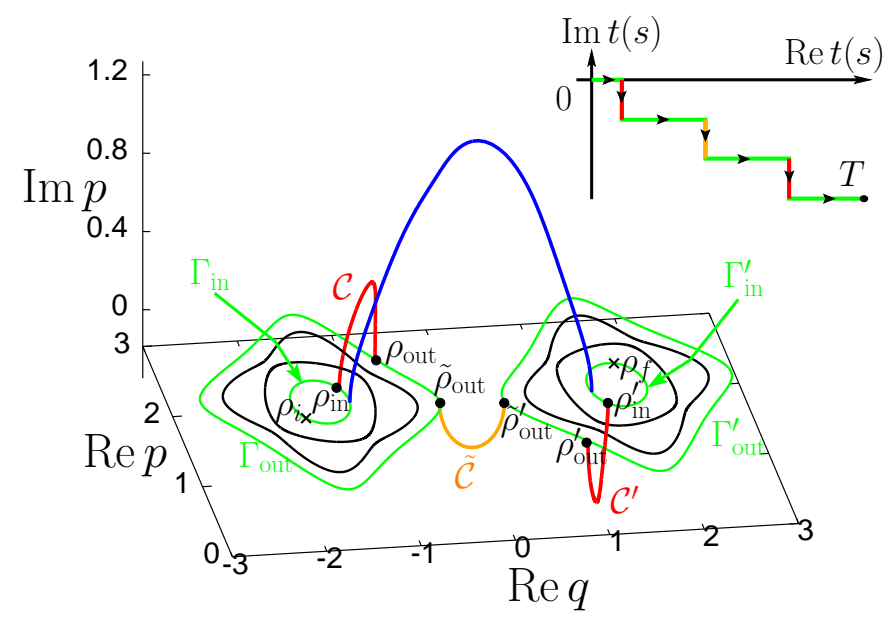

Figure 3: (Color online) Phase space of the Hamiltonian (8) (thin black lines) for $a_{1}=1, a_{2}=-0.55,|b|=0.05$, and $\phi=$ $\pi / 4$, plotted together with two different complex trajectories at energy $E \simeq 0.035$ that connect an arbitrarily chosen initial point $\rho_{i} \equiv\left(p_{i}, q_{i}\right)$ on the real torus $\Gamma_{\text {in }}$ with an arbitrarily chosen final point $\rho_{f} \equiv\left(p_{f}, q_{f}\right)$ on the symmetric counterpart $\Gamma_{\text {in }}^{\prime}$ [both tori are plotted in (light) green]. The (red and orange) arcs are half of the complex orbits $(i i) \mathcal{C}, \mathcal{C}^{\prime}$, and $(i v)$ $\tilde{\mathcal{C}}$ described in the text. Together with the connecting pieces of $\Gamma_{\text {out }}$ and $\Gamma_{\text {out }}^{\prime}$, they constitute a complex trajectory that results from the time path depicted in the upper right inset. The (dark) blue line is a complex orbit lying on a part of the complex manifold that directly connects the symmetric tori $\Gamma_{\text {in }}$ and $\Gamma_{\text {in }}^{\prime}$ (see Fig. 4). These two trajectories are plotted in a reduced three-dimensional complex phase space spanned by $(\operatorname{Re} p, \operatorname{Re} q, \operatorname{Im} p)$.

are equivalent to the set of real Hamiltonian equations

$$
\begin{aligned}
& \frac{\mathrm{d}(\operatorname{Re} q)}{\mathrm{d} s}=\frac{\partial}{\partial(\operatorname{Re} p)}\left[\operatorname{Re}\left(H \frac{\mathrm{d} t}{\mathrm{~d} s}\right)\right] ; \\
& \frac{\mathrm{d}(\operatorname{Im} q)}{\mathrm{d} s}=\frac{\partial}{\partial(-\operatorname{Im} p)}\left[\operatorname{Re}\left(H \frac{\mathrm{d} t}{\mathrm{~d} s}\right)\right] ; \\
& \frac{\mathrm{d}(\operatorname{Re} p)}{\mathrm{d} s}=-\frac{\partial}{\partial(\operatorname{Re} q)}\left[\operatorname{Re}\left(H \frac{\mathrm{d} t}{\mathrm{~d} s}\right)\right] ; \\
& \frac{\mathrm{d}(-\operatorname{Im} p)}{\mathrm{d} s}=-\frac{\partial}{\partial(\operatorname{Im} q)}\left[\operatorname{Re}\left(H \frac{\mathrm{d} t}{\mathrm{~d} s}\right)\right] ;
\end{aligned}
$$

that describes the evolution of a system with the four real canonical variables $(\operatorname{Re} p,-\operatorname{Im} p)$ and $(\operatorname{Re} q, \operatorname{Im} q)$ under the Hamiltonian $\operatorname{Re}[H(\mathrm{~d} t / \mathrm{d} s)]$.

In our case of resonance-assisted tunneling, we start from an initial point $\left(p_{i}, q_{i}\right) \equiv\left(p\left(s_{i}\right), q\left(s_{i}\right)\right)$ at time $t_{i} \equiv t\left(s_{i}\right)$ on a real inner torus $\Gamma_{\text {in }}$ inside the eye of one of the two main islands and choose a time path $t(s)$ with the shape of a descending staircase as sketched in Fig. 3. This time path is not restricted to lie all along the imaginary axis as imposed by the theory of instantons. Instead, it successively evolves along the real and imaginary directions (characterized by a real and imaginary $\mathrm{d} t / \mathrm{d} s$, respectively), such that the complex trajectory ends, after a time $T$, at the final point $\left(p_{f}, q_{f}\right)$ on the real torus

$$
\begin{aligned}
& \frac{\mathrm{d} p}{\mathrm{~d} s}=-\frac{\partial H}{\partial q} \frac{\mathrm{d} t}{\mathrm{~d} s} \\
& \frac{\mathrm{d} q}{\mathrm{~d} s}=\frac{\partial H}{\partial p} \frac{\mathrm{d} t}{\mathrm{~d} s}
\end{aligned}
$$


$\Gamma_{\text {in }}^{\prime}$ that corresponds to the counterpart of $\Gamma_{\text {in }}$ in the symmetric island. The freedom in the choice of $t(s)$ can be justified from the fact that the semiclassical contributions to tunneling, as obtained through the stationary phase approximation of the time propagator $G\left(q_{f}, q_{i}, T\right)$, arise from action integrals along complex classical trajectories that join $q_{i}$ and $q_{f}$ in a time $T$ and fulfill Eqs. (14). McLaughlin showed [48] that these integrals are independent of the time path $t(s)$ as long as no bifurcations of trajectories are encountered while deforming the path and as long as $\operatorname{Im} t(s)$ does not increase with $s$ in order to guarantee the boundedness of any intermediate evolution operator.

Tuning properly the length of the stairs as we depict in Fig. 3, complex trajectories then can be described as a continuous concatenation of pieces of the following distinct orbits:

(i) the two symmetric real periodic orbits lying on the inner tori $\Gamma_{\text {in }}$ and $\Gamma_{\text {in }}^{\prime}$ with the real energy $E$, the real period $T_{\text {in }}(E)=T_{\text {in }}^{\prime}(E)$, and the real action $S_{\text {in }}(E)=$ $S_{\text {in }}^{\prime}(E)$;

(ii) the two symmetric complex periodic orbits $\mathcal{C}$ and $\mathcal{C}^{\prime}$ with the imaginary period $\mathrm{i} T_{\mathfrak{c}}(E)=\mathrm{i} T_{\mathfrak{c}}^{\prime}(E)$ and the imaginary action $\mathrm{i} \sigma_{\mathfrak{c}}(E)=\mathrm{i} \sigma_{\mathfrak{c}}^{\prime}(E)$ with $\sigma_{\mathfrak{c}}(E)>0$, which connect the real inner tori $\Gamma_{\text {in }}$ and $\Gamma_{\text {in }}^{\prime}$ with the outer ones $\Gamma_{\text {out }}$ and $\Gamma_{\text {out }}^{\prime}$, respectively;

(iii) the two symmetric real periodic orbits lying on the outer tori $\Gamma_{\text {out }}$ and $\Gamma_{\text {out }}^{\prime}$ with the real period $T_{\text {out }}(E)=$ $T_{\text {out }}^{\prime}(E)$ and the real action $S_{\text {out }}(E)=S_{\text {out }}^{\prime}(E)>0$;

(iv) a complex periodic orbit $\tilde{\mathcal{C}}$ defined on the complex manifold that connects the outer tori $\Gamma_{\text {out }}$ and $\Gamma_{\text {out }}^{\prime}$, with the imaginary action $\mathrm{i} \tilde{\sigma}_{\mathfrak{c}}(E)$ with $\tilde{\sigma}_{\mathfrak{c}}(E)>0$ and the imaginary period $\mathrm{i} \tilde{T}_{\mathfrak{c}}(E)$.

The closed orbits $(i)-(i v)$ are geometrical objects with the property that the values of the associated actions do not depend on the choice of the canonical coordinates. Those actions are given in the $(p, q)$-representation by

$$
\begin{aligned}
S_{\text {in }}(E) & =\oint_{\Gamma_{\text {in }}} \operatorname{Re} p \mathrm{~d}(\operatorname{Re} q) ; \\
S_{\text {out }}(E) & =\oint_{\Gamma_{\text {out }}} \operatorname{Re} p \mathrm{~d}(\operatorname{Re} q) ; \\
\sigma_{\mathfrak{c}}(E) & =\oint_{\mathcal{C}}[\operatorname{Re} p \mathrm{~d}(\operatorname{Im} q)+\operatorname{Im} p \mathrm{~d}(\operatorname{Re} q)] ; \\
\tilde{\sigma}_{\mathfrak{c}}(E) & =\oint_{\tilde{\mathcal{C}}}[\operatorname{Re} p \mathrm{~d}(\operatorname{Im} q)+\operatorname{Im} p \mathrm{~d}(\operatorname{Re} q)] .
\end{aligned}
$$

For the two last actions, the contours can be continuously deformed according to Cauchy's theorem as long as no singularities of the transformation $q \mapsto p(q, E)$ are crossed (see Fig. 4).

Starting first with a portion of real time $(\mathrm{d} t / \mathrm{d} s>0)$, the trajectory evolves from the initial point $\rho_{i} \equiv\left(p_{i}, q_{i}\right)$ along the torus $\Gamma_{\text {in }}$ until it reaches a certain point $\rho_{\text {in }}$. Then the time varies along the imaginary direction $(\mathrm{id} t / \mathrm{d} s>0)$, driving the trajectory into the complex domain until it reaches again the real phase space, namely

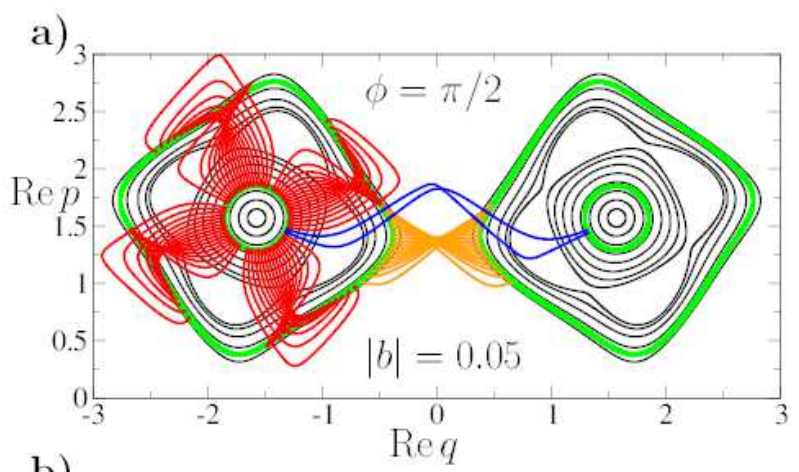

b)
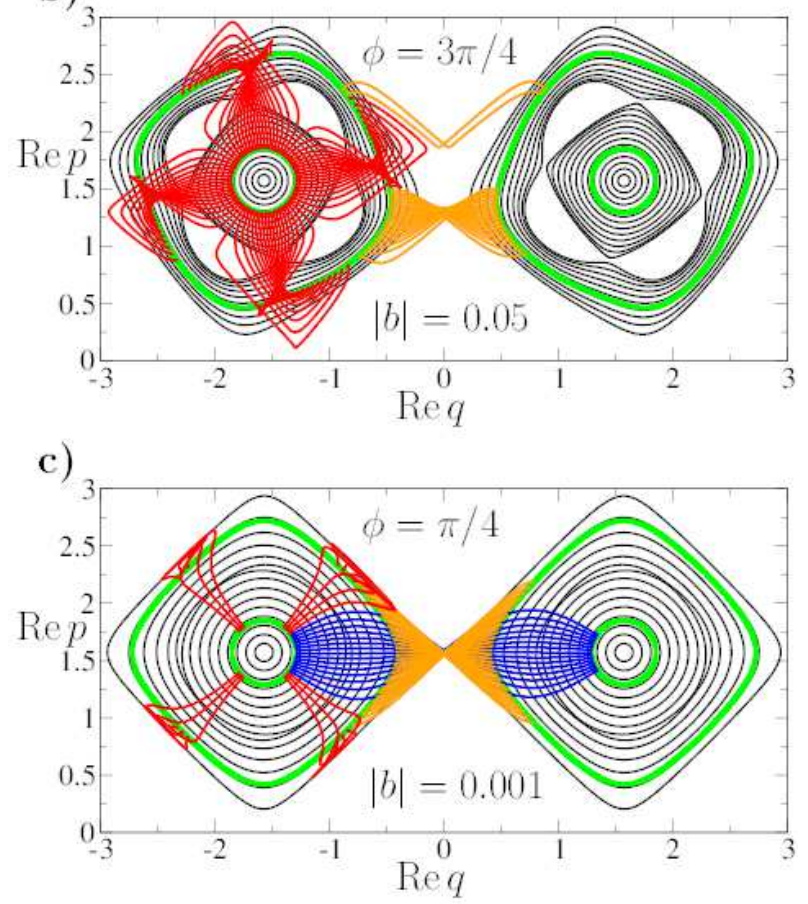

Figure 4: (Color online) Visualization of the complex manifold associated with a pair of inner real tori $\Gamma_{\text {in }}, \Gamma_{\text {in }}^{\prime}$ and the corresponding outer real tori $\Gamma_{\text {out }}, \Gamma_{\text {out }}^{\prime}$ at energy $E \simeq 0.035$ (all the four are plotted with thick green lines) for the Hamiltonian (8) with $a_{1}=1, a_{2}=-0.55$, and (a) $|b|=0.05$, $\phi=\pi / 2$; (b) $|b|=0.05, \phi=3 \pi / 4$; (c) $|b|=0.001, \phi=\pi / 4$. An exemplary set of complex orbits starting from different initial points on $\Gamma_{\text {in }}$ and $\Gamma_{\text {out }}$ is projected onto the real phase space (using the same color code as in Fig. 3), in order to illustrate the topology of this complex manifold. While there are two distinct families of (orange) complex orbits that connect the outer real tori of the two islands in (b), only the lower family (at $p<\pi / 2$ ) contributes to the tunneling process as its imaginary action is significantly smaller than the one of the upper family (at $p>\pi / 2$ ).

at the point $\rho_{\text {out }}$ on the outer torus $\Gamma_{\text {out }}$. The length of this time step is equal to $\left|T_{\mathfrak{c}}(E) / 2\right|$ such that only a half of the closed orbit $\mathcal{C}$ is followed in order to reach the outer torus. Another portion of real time is again spent to evolve on the real torus $\Gamma_{\text {out }}$ and reach the real point $\tilde{\rho}_{\text {out }}$. Then again, thanks to an imaginary time step with the length $\left|\tilde{T}_{\mathfrak{c}}(E) / 2\right|$, the trajectory evolves on the 
complex manifold and joins, after a half of a loop $\tilde{\mathcal{C}}$, the torus $\Gamma_{\text {out }}^{\prime}$ at a point $\tilde{\rho}_{\text {out }}^{\prime}$ on the other side of the main separatix delimiting the two main islands. By symmetry, this procedure is repeated to connect consecutively the real phase-space points $\rho_{\text {out }}^{\prime}$ and $\rho_{\text {in }}^{\prime}$ (for simplicity they can be taken as the symmetric partners of $\rho_{\text {out }}$ and $\rho_{\text {in }}$, respectively, though this is not necessary) and finally $\rho_{f} \equiv\left(p_{f}, q_{f}\right)$. Invoking again Cauchy's theorem, the exact location of $\rho_{\text {in }}, \rho_{\text {out }}, \rho_{\text {in }}^{\prime}$, and $\rho_{\text {out }}^{\prime}$ on the real plane is not important as long as no singularity is encountered when moving them along the corresponding real tori (see Fig. 4) [55].

We now use the different parts of the generic complex trajectory we have just described in order to split the tunneling process up into two main steps, namely (I) to cross the separatrices that delimit the resonant chains and (II) to pass over the separatrix structure that divides the two main islands.

(I) From its very construction, the global Hamiltonian (8a) can be approximated by the normal form (7) in the neighborhood of $(q, p)=( \pm \pi / 2, \pi / 2)$ and rewritten in action-angle coordinates using the canonical transformation $(p=\pi / 2+\sqrt{2 I} \cos \theta, q= \pm \pi / 2+\sqrt{2 I} \sin \theta)$ with $I>0$ and $\theta \in[0,2 \pi]$. This finally yields a modified mathematical pendulum

$$
H_{\mathrm{loc}}(I, \theta)=K_{0}+\frac{\left(I-I_{\ell: 1}\right)^{2}}{2 m_{\ell: 1}}+2 V(I) \cos \left(\ell \theta+\phi_{\ell: 1}\right)
$$

which is parametrized by coefficients that are given by the parameters of the exact global Hamiltonian:

$$
\begin{aligned}
& K_{0}=-a_{1}^{2} /\left(16 a_{2}\right), \quad I_{\ell: 1}=-a_{1} /\left(8 a_{2}\right), \\
& \phi_{\ell: 1}=\phi, \quad m_{\ell: 1}=1 /\left(8 a_{2}\right), \quad V(I)=2|b| I^{2} .
\end{aligned}
$$

This pendulum structure provides a dynamical tunnel coupling between the inner torus $\Gamma_{\text {in }}$ and the outer torus $\Gamma_{\text {out }}$.

To describe this tunneling process by means of the JWKB theory, we represent the global quasimode $|\Psi\rangle$ localized on the quantized torus $\Gamma_{\text {in }}$, which is characterized by the energy $E_{n}$ and the oscillation period $T_{\text {in }}\left(E_{n}\right)$ in angle representation as 25, Appendix C]

$$
\Psi(\theta) \simeq \frac{1}{\sqrt{T_{\mathrm{in}}\left(E_{n}\right)|\dot{\theta}|}}\left[\mathrm{e}^{\mathrm{i} \mathcal{S}_{\text {in }}\left(\theta, E_{n}\right) / \hbar}+\mathcal{A}_{\mathcal{T}} \mathrm{e}^{\mathrm{i}\left[\mathcal{S}_{\text {out }}\left(\theta, E_{n}\right) / \hbar+\mu\right]}\right]
$$

where $\dot{\theta}$ denotes the time derivative of the angle coordinate. The additional phase $\mu$ comes from the consistency with Langer connection formulas [49] and is related to the Maslov index counting the number of caustics encountered along the classical trajectory with the corresponding real action $\mathcal{S}_{\text {in }}\left(\theta, E_{n}\right)=\int_{0}^{\theta} I\left(\theta^{\prime}, E_{n}\right) \mathrm{d} \theta^{\prime}$ where $I\left(\theta^{\prime}, E_{n}\right)$ indicates the action coordinate along the torus $\Gamma_{\text {in. }}$. By construction, the action over a period, which is given by Eq. (15a), is quantized according to $S_{\text {in }}\left(E_{n}\right) \equiv \mathcal{S}_{\text {in }}\left(2 \pi, E_{n}\right)=2 \pi \hbar(n+1 / 2)$. On the other hand, the torus $\Gamma_{\text {out }}$ on the outer side of the resonance chain, with the action $S_{\text {out }}\left(E_{n}\right)$ given by Eq. (15b), is not a priori quantized and thus $S_{\text {out }}\left(E_{n}\right) /(2 \pi \hbar)-1 / 2$ is not an integer in general. The coupling amplitude $\mathcal{A}_{\mathcal{T}}$, which characterizes the tunneling-induced admixture of the component associated with $\Gamma_{\text {out }}$ to the quasimode on the inner torus $\Gamma_{\text {in }}$, then can be evaluated as [25]

$$
\mathcal{A}_{\mathcal{T}}=\frac{\mathrm{e}^{-\sigma_{\mathfrak{c}}\left(E_{n}\right) /(2 \hbar)}}{2 \sin \left[\left(S_{\text {in }}\left(E_{n}\right)-S_{\text {out }}\left(E_{n}\right)\right) /(2 \ell \hbar)\right]},
$$

where the half of the imaginary action $i \sigma_{\mathfrak{c}}\left(E_{n}\right)$ of the closed loop $\mathcal{C}$ defined by $(15 \mathrm{c}$ ) is involved.

(II) We now make use of the part of the trajectory that connects the outer torus $\Gamma_{\text {out }}$, which has the same energy $E=E_{n}$ as $\Gamma_{\text {in }}$, to its symmetric counterpart $\Gamma_{\text {out }}^{\prime}$ in the other cell. Replacing within Eq. (12) $\Sigma$ by the action of the closed orbit $\tilde{\mathcal{C}}$ and $\omega_{n}$ by the frequency $\omega_{\text {out }}$ of the outer tori, and taking into account the periodicity of our system (which gives rise to an additional factor two in the splitting formula as compared to simple doublewell tunneling), we evaluate the level splitting associated with the tori $\Gamma_{\text {out }}$ and $\Gamma_{\text {out }}^{\prime}$ at the energy $E$ due to direct tunneling across the main separatrix as

$$
\delta E(E)=\frac{2 \hbar \omega_{\text {out }}}{\pi} \mathrm{e}^{-\tilde{\sigma}_{\mathfrak{c}}(E) /(2 \hbar)} .
$$

Collecting the results (19) and (20), we obtain as a key statement of our paper the semiclassical prediction

$$
\Delta E_{n}=\left|\mathcal{A}_{\mathcal{T}}\right|^{2} \delta E\left(E_{n}\right)
$$

for the level splitting associated with the states $\left|\phi_{n}^{ \pm}\right\rangle[25]$. By symmetry, one needs to take into account twice the first step, leading to the square of the transmitted amplitude $\mathcal{A}_{\mathcal{T}}$.

\section{Comparison and discussion}

A comparison of the formula (21) with the exact splittings, which are obtained through numerical diagonalization, yields a very good agreement, as shown in Figs. 5] and 6. Peaks appear in the splitting whenever the denominator of $\mathcal{A}_{\mathcal{T}}$ vanishes, that is to say, when $\Gamma_{\text {out }}$ is a EBK quantized torus with a quantum number $\tilde{n}$ that satisfies $\tilde{n}=n+\nu \ell$ with integer $\nu$. In that case, the area $S_{\text {out }}\left(E_{n}\right)-S_{\text {in }}\left(E_{n}\right)$ enclosed by the two tori $\Gamma_{\text {in }}$ and $\Gamma_{\text {out }}$ corresponds to exactly $\nu \ell$ Planck cells of size $2 \pi \hbar$.

For finite values of the perturbation strength $|b|$, the rotation angle $\phi$ of the classical resonant chains clearly influences the splittings, which exhibit a symmetry axis at $\phi=\pi$ as shown in Fig. 6. Indeed, this behavior can be explained in terms of complex paths. Keeping in mind the local pendulum approximation (16), the real tori and the complex orbits $\mathcal{C}, \mathcal{C}^{\prime}$ that cross the resonance chains are not appreciably affected by a variation of $\phi$ which essentially corresponds to a rotation of the resonance structures in the phase space. Correspondingly, the peaks observed in the splittings remain globally at the same position when $\phi$ is varied (although they may be shifted a bit, 


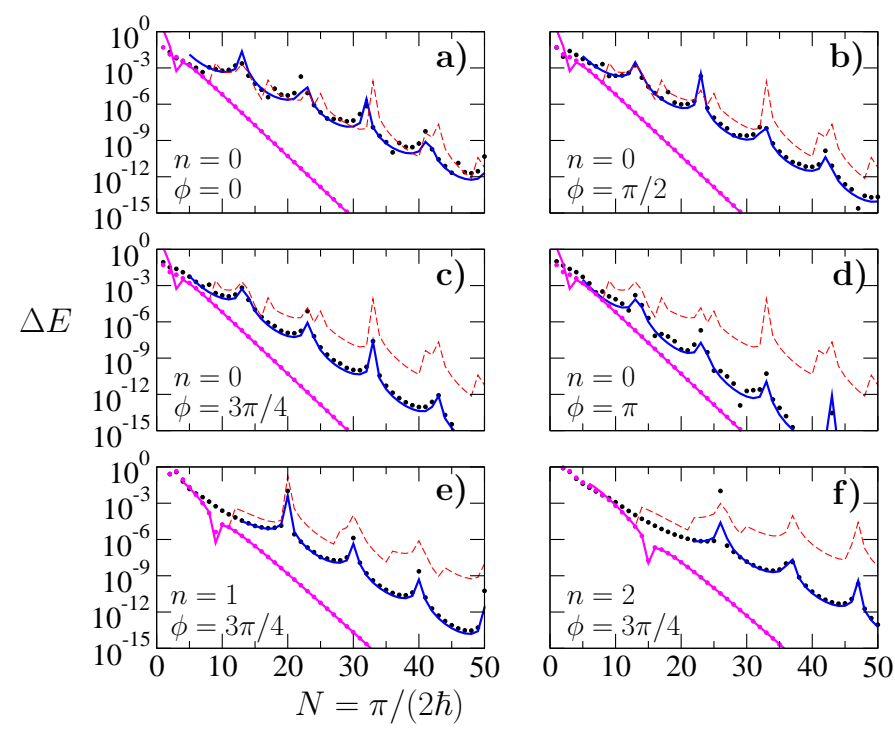

Figure 5: (Color online) Quantum and semiclassical level splittings $\Delta E_{n}$ plotted in a semilogarithmic scale versus the integer $N \equiv \pi /(2 \hbar)$ for the Hamiltonian (8) with $a_{1}=1$, $a_{2}=-0.55,|b|=0.05$ for the following phases and levels (a) $\phi=0, n=0$; (b) $\phi=\pi / 2, n=0$; (c) $\phi=3 \pi / 4, n=0$; (d) $\phi=\pi, n=0$; (e) $\phi=3 \pi / 4, n=1$; (f) $\phi=3 \pi / 4, n=2$. The (black) dots represent the exact numerical results while the (blue) solid lines show the predictions obtained by the semiclassical formula (21) [52]. The (red) dashed line is the perturbative RAT prediction obtained with the expression (22) which does not depend on $\phi$. The diagonal straight lines (plotted in magenta) correspond to the unperturbed semiclassical prediction (12) while the (magenta) dots on top show the exact splittings for the case $b=0$. The dips in the unperturbed splittings around $N \simeq 3,9,15$, respectively, for $n=0,1,2$ arise when the unperturbed quantized torus is located right on the crown of the volcanos. In that case, the classical frequency of the torus vanishes and, as a consequence, Eq. (12) predicts a vanishing level splitting.

see the upper right panel of Fig. (6). On the other hand, the imaginary action of the orbit $\tilde{\mathcal{C}}$ that crosses the main separatrix between the islands is significantly modified under variation of $\phi$. As one can indeed see in Fig. 4 the complex "bridge" (plotted in orange) that connects the two outer symmetric tori of the main islands is shifted farther away from the horizontal symmetry axis as $\phi$ is increased, which is naturally accompanied by an increase of the corresponding imaginary action. This is responsible for the drastic decrease of the splitting (by three orders of magnitude for $N \sim 25$ as is seen in Fig. (6) as $\phi$ is varied from 0 to $\pi$.

It is instructive to compare the exact splittings and their semiclassical prediction also with the perturbative theory of resonance-assisted tunneling (RAT), which was first introduced for 1D time-periodic Hamiltonians in the quasi-integrable regime [24, 25] and later extended to mixed regular-chaotic systems [26, 28, 50]. Following the derivation described in the Appendix $\mathrm{A}$, the level splitting associated with the eigenstates $\left|\phi_{n}^{ \pm}\right\rangle$of the Hamilto-

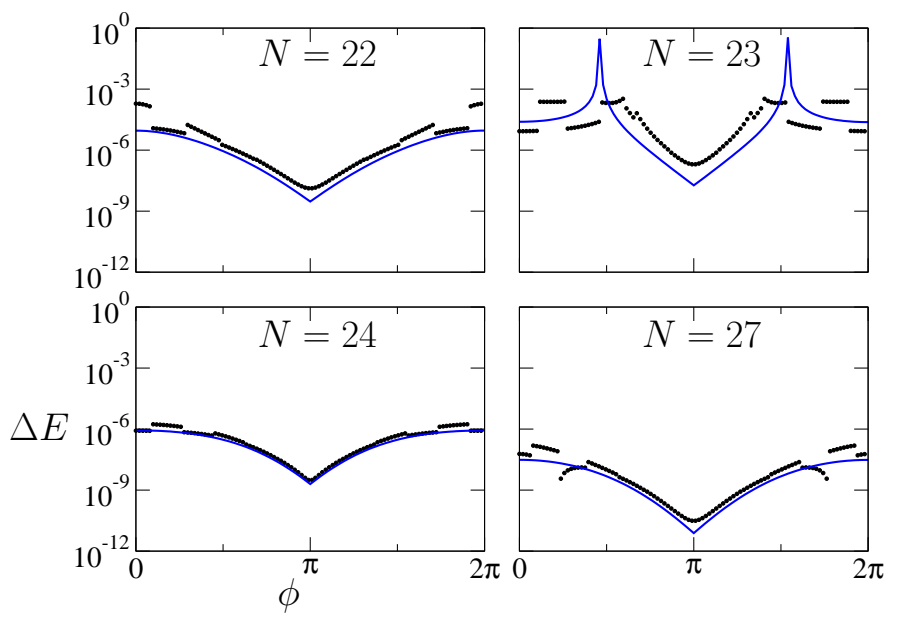

Figure 6: (Color online) Quantum and semiclassical level splittings $\Delta E_{0}$ associated with the eigenstates that are most strongly localized on the centres of the four symmetric islands, for the Hamiltonian (8) with $a_{1}=1, a_{2}=-0.55,|b|=0.05$. The splittings are plotted versus the phase $\phi$ for different values of $N \equiv \pi /(2 \hbar)=22$ (upper left panel), 23 (upper right panel), 24 (lower left panel),and 27 (lower right panel). The (black) dots represent the exact numerical results while the (blue) solid lines are the semiclassical predictions obtained from Eq. (21).

nian is given by

$$
\Delta E_{n}=\Delta E_{n}^{(0)}+\sum_{k>0}^{k_{c}}\left|B_{n, k \ell}\right|^{2} \Delta E_{n+k \ell}^{(0)},
$$

with $\ell \equiv 4$ and

$$
\begin{aligned}
B_{n, k \ell} & =\prod_{p=1}^{k} \frac{A_{n+p \ell, n+(p-1) \ell}}{E_{n}^{(0)}-E_{n+p \ell}^{(0)}} ; \\
A_{n+p \ell, n+(p-1) \ell} & =2|b| \mathrm{e}^{\mathrm{i} \phi} \hbar^{p \ell / 2} \sqrt{\frac{(n+p \ell) !}{[n+(p-1) \ell] !}},
\end{aligned}
$$

where the $E_{n+p \ell}^{(0)}$ denote the unperturbed energies (i.e. for $b=0)$ and

$$
\Delta E_{n+k \ell}^{(0)} \simeq \frac{2 \hbar \omega_{n+k \ell}^{(0)}}{\pi} \mathrm{e}^{-\sigma_{n+k \ell}^{(0)} / 2 \hbar}
$$

the unperturbed splittings. The latter are determined through a numerical evaluation of the oscillation frequencies $\omega_{n+k \ell}^{(0)}$ and the imaginary actions $\sigma_{n+k \ell}^{(0)}$ associated with the unperturbed invariant tori at energy $E_{0}$. Using the quadratic approximation (A3), one notices that the denominators of the coefficients (23) are proportional to the quantized action variables of the unperturbed system: $\left(E_{n}^{(0)}-E_{n+p \ell}^{(0)}\right) \propto\left(I_{n}-I_{n+p \ell}\right)\left(I_{n}+I_{n+p \ell}-2 I_{\ell: s}\right)$. The coupling between the unperturbed states $|n\rangle$ and $|n+p \ell\rangle$ is therefore maximized when the quantized tori $I_{n}$ and 


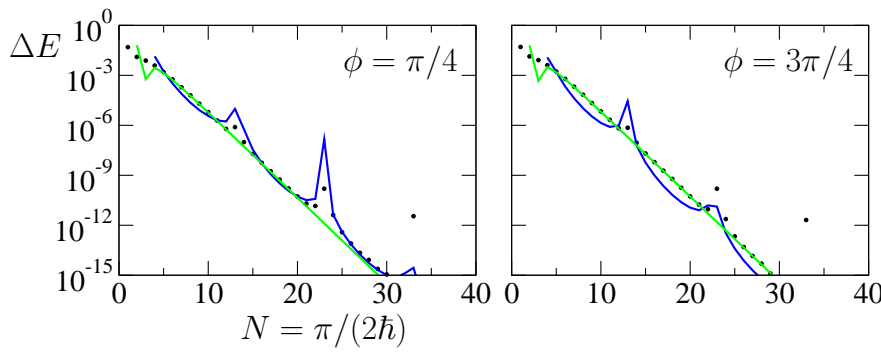

Figure 7: (Color online) Quantum and semiclassical level splittings $\Delta E_{0}$ associated with the eigenstates that are most strongly localized on the centers of the four symmetric islands, for the Hamiltonian (8a) with the parameters $a_{1}=1$, $a_{2}=-0.55$ and $|b|=0.001$, plotted versus $N \equiv \pi /(2 \hbar)$ for $\phi=\pi / 4$ (left panel) and $\phi=3 \pi / 4$ (right panel). The (black) dots represent the exact results, the dark (blue) solid lines correspond to the resonance-assisted semiclassical splittings obtained with the semiclassical formula (21), and the light (green) straight lines show the direct splittings given by Eq. (26). The effect of the resonance is significantly reduced as compared to Fig. 5, and the splittings no longer display systematic variations as a function of the rotation angle $\phi$.

$I_{n+p \ell}$ are symmetrically located with respect to the resonant island chain which is approximately localized at $I_{\ell: s}$. This then leads to significant local enhancements of the splittings.

However, the above formulation of the perturbative RAT theory does not account for a modification of the imaginary actions $\sigma_{n+k \ell}^{(0)}$ due to the presence of the resonance chain. While this modification can be safely neglected in generic near-integrable systems which generally exhibit perturbatively small resonance chains [24, 25], it does matter in our special case of integrable resonance-assisted tunneling with macroscopically large resonance islands as we pointed out above. We are therefore already beyond the perturbative regime. This is clearly seen in Fig. [5] Even though the perturbative RAT predictions are, by coincidence, still in approximate agreement with the exact splittings for $\phi=0$ [56], they drastically overestimate the latter for $\phi=\pi$.

Let us finally discuss the interplay of resonanceassisted tunneling with direct tunneling in the deep perturbative regime. With decreasing $|b|$, the splittings are less and less sensitive to a variation of the phase $\phi$ and the resonance peaks become less and less pronounced. Direct tunneling becomes the dominant mechanism in the limit $|b| \rightarrow 0$, and the splittings display a purely exponential decrease with $1 / \hbar$, which can be evaluated as

$$
\delta E_{n}^{(d)}=\frac{2 \hbar \omega_{\text {in }}}{\pi} \mathrm{e}^{-\Sigma\left(E_{n}\right) /(2 \hbar)}
$$

where $\mathrm{i} \Sigma\left(E_{n}\right)$ [with $\Sigma\left(E_{n}\right)>0$ ] is the imaginary action of the complex manifold (plotted in blue in Fig. 4) that directly connects the real tori $\Gamma_{\text {in }}$ and $\Gamma_{\text {in }}^{\prime}$, and $\omega_{\text {in }}$ is the frequency of those two tori. In the limit $|b| \rightarrow 0$, the action i $\Sigma\left(E_{n}\right)$ coincides with the unpertubed one i $\Sigma^{(0)}\left(E_{n}\right)$ and, hence, $\delta E_{n}^{(d)}$ approaches the unperturbed splittings $\Delta E_{n}^{(0)}$.

In the context of the RAT theory, Löck et al [28] developed a quantitative criterion providing, for a given strength of the perturbation, the characteristic value $\hbar_{\text {res }}$ of Planck's constant that separates the "direct" regime $\left(\hbar>\hbar_{\text {res }}\right)$ in which direct tunneling dominates from the "resonance-assisted" regime $\left(\hbar<\hbar_{\text {res }}\right)$ in which the relevant mechanism is resonance-assisted tunneling. This criterion is given by the equality

$$
\frac{\left(\Delta \mathcal{A}_{\ell: s}\right)^{2}}{\ell \hbar_{\mathrm{res}} \mathcal{A}_{\ell: s}} \sqrt{\frac{\Delta E_{n+\ell}^{(0)}\left(\hbar_{\mathrm{res}}\right)}{\Delta E_{n}^{(0)}\left(\hbar_{\mathrm{res}}\right)}} \frac{1}{\left(\hbar_{\mathrm{res}} / \hbar_{\mathrm{peak}}\right)-1}=\frac{256}{\pi},
$$

where $\Delta \mathcal{A}_{\ell: s}=16 \sqrt{2 m_{\ell: s} V_{\ell: s}}$ is the area covered by the island chain [26] as derived from the pendulum approximation $(16), \mathcal{A}_{\ell: s}=2 \pi I_{\ell: s}$ is the area enclosed by the approximate resonant torus of the unperturbed system, and $\hbar_{\text {peak }}$ is the value of Planck's constant at which the first peak in the splittings $\Delta E_{n}$ appears. Using $\hbar_{\text {peak }} \equiv \pi /\left(2 N_{\text {peak }}\right)$ with $N_{\text {peak }} \simeq 13.5(, 20,26.25)$ for the level $n=0(, 1,2)$, as extracted from Figs. 5 and 7 , we obtain $N_{\text {res }} \equiv \pi /\left(2 \hbar_{\text {res }}\right) \simeq 9(, 12,14)$ for $|b|=0.05$ and $N_{\text {res }} \equiv \pi /\left(2 \hbar_{\text {res }}\right) \simeq 13$ for $|b|=0.001$, which is in rather good agreement with Figs. 5 and 7 , respectively.

\section{CONCLUSIONS}

In summary, we discussed a semiclassical theory of resonance-assisted tunneling in integrable systems, which is based on the analytic continuation of the invariant classical tori of the system to the complex domain. To this end, we showed how to construct a class of 1D integrable Hamiltonians, based on the normal form theory, that exhibit islands of bounded motion surrounded by chains that mimic the resonance structures arising in Poincaré sections of nonintegrable systems. We then studied tunneling between two symmetric islands in such integrable systems. Our semiclassical theory, which is essentially expressed by Eqs. (19) and (21), is found to reproduce the numerically computed tunneling splittings with rather good accuracy. In contrast to the standard implementation of the RAT theory which is based on quantum perturbation theory, Eqs. (19) and (21) provide reliable predictions of level splittings also in the nonperturbative regime characterized by rather well-developed resonance island chains. In that case, a rotation of the resonance chain with respect to the main separatrix of the system may have a significant impact on the tunneling rates of the system, due to the associated displacement of the invariant manifolds that cross the separatrix and govern direct tunneling outside the resonance chain.

Even though a full derivation of our semiclassical theory is not presented here, the similarity of Eqs. (19) and (21) with the analytical expression for the level splittings in a triple-well potential derived in Ref. [11] 
[see Eq. (66) there] suggests that our main result (21) could eventually be derived by adapting the semiclassical framework developed in Ref. 11] [see Eq. (40) there] to our case of resonance-assisted tunneling through island chains (although this latter case topologically differs markedly from the triple-well system). Indeed, we expect that the resonance peaks observed in the splittings arise due to constructive Fabry-Pérot type interferences between topologically distinct complex trajectories that connect the two islands via the periodic orbits introduced in Sec. IIIB which are frequented with distinct repetitions. The main difficulty in establishing such a semiclassical framework in the spirit of Ref. [11] comes from the selection of the class of complex orbits that brings the main contribution. For a Hamiltonian of the form (1), the restriction to complex periodic orbits along which one canonical variable remains purely real provides a useful help. However, an analogous restrictive criterion is not known to us for the resonance-assisted tunneling problem under consideration.

Through recursive application of the basic principle of resonance-assisted tunneling, one may expect to derive a generalized semiclassical expression for level splittings between islands that contain $R>1$ different $\left(\ell_{r}: s_{r}\right)$ island chains for $r=1, \ldots, R$. Such a semiclassical expression is expected to be of the form

$$
\Delta E_{n}=\left[\prod_{r=1}^{R}\left|\mathcal{A}_{\mathcal{T}}^{\left(\ell_{r}: s_{r}\right)}(\hbar)\right|^{2}\right] \delta E\left(E_{n}\right)
$$

where $\delta E\left(E_{n}\right)$ is the direct splitting (20) associated with the outermost torus involved in this multiresonance transition process. In the perturbative regime, the individual coupling amplitudes $\mathcal{A}_{\mathcal{T}}^{\left(\ell_{r}: s_{r}\right)}(\hbar)$ can be approximately evaluated using a local pendulum approximation for each $\left(\ell_{r}: s_{r}\right)$ resonance chain (see also Eq. (76) in Ref. 25]). However, there is no guarantee that this approach remains valid in the presence of nonperturbatively large resonance chains. A careful investigation of the complex manifolds will be required in that case in order to determine which type of complex paths are relevant depending on the relative size of the chains with respect to Planck's constant.

Finally, our theory may provide a useful starting point for developing a quantitative semiclassical description of tunneling also in nonintegrable systems that exhibit a mixed regular-chaotic phase space structure. In analogy with the perturbative RAT study of Ref. [28], resonanceassisted transitions will, in that case, have to be combined with direct regular-to-chaotic tunneling [30] for which a fully semiclassical theory in terms of complex paths was recently presented in Ref. [51]. It seems straightforward to incorporate the effect of nonlinear resonances into this latter semiclassical framework of Ref. [51], in order to extend its applicability to the deep semiclassical regime in which resonances generically play a role.

\section{Acknowledgments}

This work was financially supported by the BayerischFranzösisches Hochschulzentrum (BFHZ-CCFUB), by the Deutsche Forschungsgemeinschaft in the framework of the DFG Forschergruppe FOR760, and by the Advanced Study Group (ASG) "Towards a Semiclassical Theory of Dynamical Tunneling" which took place at the Max-Planck-Institut für Physik Komplexer Systeme (MPIPKS). We thank the members and invited speakers of the ASG, in particular Roland Ketzmerick, Steffen Löck, Normann Mertig, Akira Shudo, and Denis Ullmo, for useful discussions.

\section{Appendix A: Theory of resonance-assisted tunneling for $1 \mathrm{D}$ integrable systems}

In this appendix, we review the main steps of the perturbative approach to describe resonance-assisted tunneling (RAT) applied to the simple case of one-dimensional integrable Hamiltonians. In analogy with the procedure described in Ref. 29] (see also Refs. 24, 25, 28]), we start with a 1D time-independent Hamiltonian $H(p, q)$ (which is assumed to be an analytical function in $p$ and $q)$ that exhibits in the phase space two main symmetric regions, each of them surrounded by one $\ell: s$ resonant island chain. Approximate action-angle variables $(I, \theta)$, which result from $(p, q)$ via a canonical transformation, can be defined locally within each of the two regions. Their time evolution is governed by a Hamiltonian of the form

$$
H(I, \theta)=H_{0}(I)+V(I, \theta)
$$

[for the sake of simplicity, we shall keep the same notation $H(\cdot, \cdot)$ for both the $(p, q)$ and the $(I, \theta)$ representations], where the angle-dependent perturbation $V(I, \theta)$ is responsible for the generation of the resonant chain. We now expand the perturbation as a Fourier series

$$
V(I, \theta)=\sum_{j=1}^{\infty} 2 V_{j}(I) \cos \left(j \ell \theta+\phi_{j}\right),
$$

and perform a harmonic approximation of the angleindependent part of the Hamiltonian near the resonant chain,

$$
H_{0}(I) \simeq H_{0}\left(I_{\ell: s}\right)+\frac{\left(I-I_{\ell: s}\right)^{2}}{2 m_{\ell: s}}+\mathrm{O}\left[\left(I-I_{\ell: s}\right)^{3}\right],
$$

where $I_{\ell: s}$ is the action variable at the resonance and $1 / m_{\ell: s} \equiv \mathrm{d}^{2} H_{0} / \mathrm{d} I^{2}$ at $I=I_{\ell: s}$. By definition, the frequency of oscillations $\Omega \equiv \mathrm{d} H_{0} / \mathrm{d} I$ vanishes at the resonance. Combining Eqs. A2 and (A3) [and omitting the constant $\left.H_{0}\left(I_{\ell: s}\right)\right], H(I, \theta)$ is reduced to a modified generalized pendulum of the form

$$
H_{\text {pend }}(I, \theta) \stackrel{\text { def }}{=} \frac{\left(I-I_{\ell: s}\right)^{2}}{2 m_{\ell: s}}+\sum_{j=1}^{\infty} 2 V_{j}(I) \cos \left(j \ell \theta+\phi_{j}\right) \text {. }
$$


Treating $V(I, \theta)$ as a small perturbation, one can now apply the time-independent quantum perturbation theory. Using the eigenstates $|n\rangle$ (with $n \in \mathbb{N}_{0}$ ) of the operator $\hat{I}=-\mathrm{i} \hbar \partial / \partial \hat{\theta}$ which fulfill

$$
\begin{aligned}
\hat{I}|n\rangle & =I_{n}|n\rangle=\hbar(n+1 / 2)|n\rangle, \\
H_{0}(\hat{I})|n\rangle & =E_{n}^{(0)}|n\rangle,
\end{aligned}
$$

one can notice that $\hat{V}(\hat{I}, \hat{\theta})$ induces only couplings between the unperturbed states $|n\rangle$ and $|n+j \ell\rangle$ through the matrix elements

$$
A_{n+j \ell, n} \stackrel{\text { def }}{=}\left\langle n+j \ell\left|H_{\text {pend }}(\hat{I}, \hat{\theta})\right| n\right\rangle .
$$

In this basis, the true eigenstates of $H_{\text {pend }}(\hat{I}, \hat{\theta})$ can be approximated by the following expression

$$
\begin{aligned}
& \left|\Psi_{n}\right\rangle \simeq|n\rangle+\sum_{k} \frac{A_{n+k \ell, n}}{E_{n}^{(0)}-E_{n+k \ell}^{(0)}}|n+k \ell\rangle \\
& \quad+\sum_{k, k^{\prime}} \frac{A_{n+k \ell, n+k^{\prime} \ell}}{E_{n}^{(0)}-E_{n+k \ell}^{(0)}} \frac{A_{n+k^{\prime} \ell, n}}{E_{n}^{(0)}-E_{n+k^{\prime} \ell}^{(0)}}|n+k \ell\rangle+\cdots .
\end{aligned}
$$

As the perturbation is analytic, one can safely assume that the coefficients $V_{j}(I)$ decrease exponentially with $j$ [25]. This property is used in Ref. [25] to show that the coupling between $|n\rangle$ and $|n+k \ell\rangle$ via the $k$-steps process involving the matrix elements $A_{n+\ell, n}, A_{n+\ell, n+2 \ell}$, $\ldots, A_{n+(k-1) \ell, n+k \ell}$ is generally much stronger than the direct coupling via the matrix element $A_{n+k \ell, n}$. This allows us thus to retain only the first term $j=1$ in Eq. (A4) and to reduce the Hamiltonian to a modified mathematical pendulum

$$
H(I, \theta) \simeq \frac{\left(I-I_{\ell: s}\right)^{2}}{2 m_{\ell: s}}+2 V_{1}(I) \cos \left(\ell \theta+\phi_{1}\right)
$$

The perturbed eigenstates thus can be expressed as

$$
\left|\Psi_{n}\right\rangle \simeq|n\rangle+\sum_{k>0} B_{n, k \ell}|n+k \ell\rangle
$$

with

$$
B_{n, k \ell}=\prod_{p=1}^{k} \frac{A_{n+p \ell, n+(p-1) \ell}}{E_{n}^{(0)}-E_{n+p \ell}^{(0)}}
$$

The next step is to evaluate the coefficients $V_{j}(I)$. Using the analyticity of $H(p, q)$, one can define a local canonical transformation $(p, q) \mapsto(P, Q)$ such that $\exp ( \pm \mathrm{i} j \theta)=[(Q \mp \mathrm{i} P) / \sqrt{2 I}]^{j}$. In the new coordinates, the perturbation (A2) reads

$V(P, Q)=\sum_{j=1}^{\infty} \frac{V_{j}(I)}{(2 I)^{j \ell / 2}}\left[(Q-\mathrm{i} P)^{j \ell} \mathrm{e}^{\mathrm{i} \phi_{j}}+(Q+\mathrm{i} P)^{j \ell} \mathrm{e}^{-\mathrm{i} \phi_{j}}\right]$, where the $V_{j}(I)$ must be at least of order $I^{j \ell / 2}$ to be consistent with the normal form theory described in Sec. IA Making the assumption that $V_{j}(I)=v_{j} I^{j \ell / 2}$, we obtain

$$
V(P, Q)=\sum_{j=1}^{\infty} \frac{v_{j}}{2^{j \ell / 2}}\left[(Q-\mathrm{i} P)^{j \ell} \mathrm{e}^{\mathrm{i} \phi_{j}}+(Q+\mathrm{i} P)^{j \ell} \mathrm{e}^{-\mathrm{i} \phi_{j}}\right] .
$$

The corresponding quantum operators $(\hat{P}, \hat{Q})$ can be expressed in terms of the ladder operators $\left(\hat{a}, \hat{a}^{\dagger}\right)$ that are associated with the eigenstates $|n\rangle$ through the relations

$$
\begin{aligned}
\hat{a} & =\frac{1}{\sqrt{2}}(\hat{Q}+\mathrm{i} \hat{P}) ; \\
\hat{a}^{\dagger} & =\frac{1}{\sqrt{2}}(\hat{Q}-\mathrm{i} \hat{P}) .
\end{aligned}
$$

Using these relations in the quantization of the perturbation (A13), the matrix elements (A7) become finally

$$
A_{n+j \ell, n}=v_{j} \hbar^{j \ell / 2} \mathrm{e}^{\mathrm{i} \phi_{j}} \sqrt{\frac{(n+j \ell) !}{n !}} .
$$

In our case, this expression does not represent an approximation since the action dependence $V_{j}(I)=v_{j} I^{j \ell / 2}$ of the perturbation is, as shown Sec. IIB imposed by the construction procedure of the Hamiltonian through the framework of normal forms.

Coming back to the original Hamiltonian $H(\hat{p}, \hat{q})$, one can define in each symmetric well two distinct quasimodes $\left|\Psi_{n}^{L}\right\rangle$ and $\left|\Psi_{n}^{R}\right\rangle$ that are constructed on the symmetric tori with the energy $E_{n}$. This energetic degeneracy is lifted due to tunneling between the wells, and the level splitting $\Delta E_{n}$ is evaluated as the coupling matrix element between the quasimodes: $\Delta E_{n}=$ $\left\langle\Psi_{n}^{L}|H(\hat{p}, \hat{q})| \Psi_{n}^{R}\right\rangle$. Using Eqs. (A10), (A11) and (A16), the splitting for an arbitrary level can finally be written as

$$
\Delta E_{n}=\Delta E_{n}^{(0)}+\sum_{k=1}^{k_{c}}\left|B_{n, k \ell}\right|^{2} \Delta E_{n+k \ell}^{(0)}
$$

For a standard double well system, we would have

$$
\Delta E_{n+k \ell}^{(0)} \simeq \frac{\hbar \omega_{n+k \ell}^{(0)}}{\pi} \mathrm{e}^{-\sigma_{n+k \ell}^{(0)} / \hbar}
$$

as the splitting for the $(n+k \ell)$-th doublet of the unperturbed system $H_{0}(\hat{p}, \hat{q})$, while an additional factor 2 arises on the right-hand side of Eq. A18 in our case of a periodic array of wells. $\sigma_{n+k \ell}^{(0)}$ is the action of the instanton-like trajectory connecting the two symmetric tori with energy $E_{n+k \ell}^{(0)}$, and $\omega_{n+k \ell}^{(0)}$ is the corresponding oscillation frequency. By construction, the RAT process may only couple quasimodes that are localized within the same region of regular oscillations. The index $k_{c} \ell$ labels the most highly excited state that can be involved in a perturbative coupling scheme starting from the $n$-th excited state. Defining by $\mathcal{A}$ the area one of those regions 
(which in Fig. 2 corresponds to the area enclosed by the separatrices within each cell), we have

$$
k_{c}=\left\lfloor\frac{1}{\ell}\left(\frac{\mathcal{A}}{2 \pi \hbar}-\frac{2 n+1}{2}\right)\right\rfloor,
$$

where $\lfloor\cdot\rfloor$ stands for the integer part of a real number.
[1] M. J. Davis and E. J. Heller, J. Chem. Phys. 75, 246 (1981).

[2] N. T. Maitra and E. J. Heller, Phys. Rev. A 54, 4763 (1996).

[3] M. V. Berry and K. E. Mount, Reports on Progress in Physics 35, 315 (1972).

[4] R. Balian and C. Bloch, Ann. Phys. 85, 514 (1974).

[5] N. T. Maitra and E. J. Heller, Phys. Rev. Lett. 78, 3035 (1997).

[6] K. F. Freed, J. Chem. Phys. 56, 692 (1972).

[7] T. F. George and W. H. Miller, J. Chem. Phys. 56, 5722 (1972).

[8] W. H. Miller, J. Chem. Phys. 61, 1823 (1974).

[9] S. Coleman, Aspects of Symmetry (selected Erice lectures) (Cambridge University Press, Cambridge, 1985).

[10] Instantons in Gauge Theories Advanced Series in Mathematical Physics, Vol. 5, edited by M. A. Shifman (World Scientific, Singapore, 1994).

[11] J. Le Deunff and A. Mouchet, Phys. Rev. E 81, 046205 (2010).

[12] D. Bohm, Quantum Theory (Prentice Hall, Englewood Cliffs, NJ, 1951).

[13] H. Cruz, A. Hernandez-Cabrera, and A. Munoz, Semiconductor Science and Technology 6, 218 (1991).

[14] Y. Zohta, Phys. Rev. B 41, 7879 (1990).

[15] J. Le Deunff, O. Brodier, and A. Mouchet, Eur. J. Phys. 33, 1771 (2012).

[16] W. A. Lin and L. E. Ballentine, Phys. Rev. A 45, 3637 (1992).

[17] O. Bohigas, S. Tomsovic, and D. Ullmo, Physics Reports 223, 43 (1993).

[18] J. M. Greene and I. C. Percival, Physica D 3, 530 (1981).

[19] A. Shudo and K. S. Ikeda, Phys. Rev. Lett. 74, 682 (1995).

[20] A. Shudo, Y. Ishii, and K. S. Ikeda, J. Phys. A: Math. Gen. 42, 265101 (2009).

[21] S. C. Creagh and N. D. Whelan, Phys. Rev. Lett. 77, 4975 (1996).

[22] S. Tomsovic and D. Ullmo, Phys. Rev. E 50, 145 (1994).

[23] F. Leyvraz and D. Ullmo, J. Phys. A: Math. and Gen. 29, 2529 (1996).

[24] O. Brodier, P. Schlagheck, and D. Ullmo, Phys. Rev. Lett. 87, 064101 (2001).

[25] O. Brodier, P. Schlagheck, and D. Ullmo, Ann. Phys. 300, 88 (2002).

[26] C. Eltschka and P. Schlagheck, Phys. Rev. Lett. 94, 014101 (2005).

[27] A. Mouchet, C. Eltschka, and P. Schlagheck, Phys. Rev. E 74, 026211 (2006).

[28] S. Löck, A. Bäcker, R. Ketzmerick, and P. Schlagheck, Phys. Rev. Lett. 104, 114101 (2010).

[29] P. Schlagheck, A. Mouchet, and D. Ullmo, in Dynamical Tunneling: Theory and Experiment, edited by S. Keshavamurthy and P. Schlagheck (Taylor \& Francis/CRC, Boca Raton, FL, 2011).
[30] A. Bäcker, R. Ketzmerick, S. Löck, and L. Schilling, Phys. Rev. Lett. 100, 104101 (2008).

[31] A. Bäcker, R. Ketzmerick, and S. Löck, Phys. Rev. E 82, 056208 (2010).

[32] V. I. Arnold, Mathematical Methods of Classical Mechanics (Graduate Texts in Mathematics), 2nd ed. (SpringerVerlag, New York, 1989).

[33] A. M. Ozorio de Almeida, Hamiltonian Systems: Chaos and Quantization (Cambridge University Press, New York, 1988).

[34] G. D. Birkhoff, Dynamical Systems (American Mathematical Society Colloquium Publications, New York, 1927).

[35] F. G. Gustavson, The Astronomical Journal 71, 670 (1966).

[36] R. T. Swimm and J. B. Delos, J. Chem. Phys. 71, 1706 (1979).

[37] B. Eckhardt, J. Phys. A: Math. Gen. 19, 2961 (1986).

[38] P. Leboeuf and A. Mouchet, Ann. Phys. 275, 54 (1999).

[39] K. Husimi, Proc. Phys. Math. Soc. Jpn. 22, 264 (1940).

[40] K. Takahashi and N. Saitô, Phys. Rev. Lett. 55, 645 (1985).

[41] G. Torres-Vega and J. H. Frederick, J. Chem. Phys. 93, 8862 (1990).

[42] H. Weyl, The Theory of Groups and Quantum Mechanics (Dover, New York, 1950).

[43] J. R. Shewell, American Journal of Physics 27, 16 (1959).

[44] M. Wilkinson, Physica D 21, 341 (1986).

[45] S. C. Creagh, in Tunneling in Complex Systems, Proceedings from the Institute for Nuclear Theory, edited by S. Tomsovic (World Scientific, Singapore, 1997), Vol. 5, pp. $35-100$.

[46] L. D. Landau and E. M. Lifshitz, Quantum Mechanics: Non-Relativistic Theory, Course of Theoretical Physics, Vol. 3, 3rd ed. (Pergamon Press, Oxford, 1977).

[47] A. Garg, American Journal of Physics 68, 430 (2000).

[48] D. W. McLaughlin, J. Math. Phys. 13, 1099 (1972).

[49] R. E. Langer, Physical Review 51, 669 (1937).

[50] S. Löck, A. Bäcker, and R. Ketzmerick, Phys. Rev. E 85, 016210 (2012).

[51] N. Mertig, S. Löck, A. Bäcker, R. Ketzmerick, and A. Shudo, Europhys. Lett. 102, 10005 (2013).

[52] The latter can be properly evaluated as soon as $N>$ $N_{\mathrm{s}}$ where $N_{\mathrm{s}}$ corresponds to the value of $\hbar$ at which the initial state $\left|\phi_{n}\right\rangle$ is semiclassically associated with the inner separatrix structure of the island chain.

[53] The normal form of third order exhibits only one chain of three unstable points as shown in [38] and therefore is not representative of what occurs for higher orders.

[54] Technically this requires that the dimension $2 \pi / \hbar$ of the Hilbert space is an integer multiple of 4 . Therefore, in the following we will restrict ourselves to the case where $N=$ $\pi / 2 \hbar$ is integer.

[55] In principle, one would even be allowed to follow a time path where $\operatorname{Re} t(s)$ decreases with $s$ somewhere along the 
path. However, as the final time $T$ is fixed in the semiclassical framework developed in Ref. [11], one would, in this latter case, have to compensate this backward motion by the exactly same forward contribution on the symmetric torus in order to reach $T$. The real and imaginary action integrals along this time path would therefore be exactly the same as for the time path shown in Fig. 3 .

[56] We attribute this coincidence to the fact that the complex orbit $\tilde{\mathcal{C}}$ connecting the two symmetric outer tori can, for $\phi=0$, still be defined along the horizontal symmetry axis $\operatorname{Re} p=\pi / 2$ as in the unperturbed case $b=0$. 